ORIGINAL ARTICLE

\title{
Association of Adverse Effects of Red Meat Consumption with Liver Cirrhosis
}

GULSHAD WAGAN ${ }^{1}$, ABDULLAH KHILJI ${ }^{2}$, KALEEM SHEIKH ${ }^{3}$, SYED SOHAIL ABBAS NAQVI ${ }^{4}$, IMTIAZ WAGAN ${ }^{5}$, SYEDA ABIYA AMBER NAQVI ${ }^{6}$

${ }^{1}$ Assistant professor Anatomy, Bilawal Medical College, Jamshoro

${ }^{2}$ Assistant Professor Anatomy, Khairpur Medical College, Khairpur Mir's

${ }^{3}$ PHC Mangement Trainer, PHDC Jamshoro

${ }^{4}$ Assistant Professor Pathology, Khairpur Medical College, Khairpur Mir's

${ }^{5}$ Professor of Anatomy, Dow University of Health Science, Karachi

${ }^{6}$ Senior Lecturer, Sir Syed College of Medical Sciences for Girls, Karachi

Corresponding author: Dr. Gulshad Wagan, Email: aishakaleem497@gmail.com, Contact No.0333-2627204

\begin{abstract}
Objective: To determine the association of adverse outcomes in term of mortality in patients with cirrhosis and red meat consumption.

Study Design: Cross-sectional

Place and Duration: Medicine Department of Liaquat University Medical Hospital Jamshoro and Civil Hospital Khairpur Mir's for six months duration from December 2019 to November 2020.

Methodology: Total 120 patients of both genders with chronic liver disease were enrolled in this study. Patients were categorized in to two groups. Group A (red meat consumption $>60 \mathrm{gm} /$ day) and group B (red meat consumption $<60 \mathrm{gm} /$ day). Outcomes in term of mortality between both groups were examined. All the data was analyzed by SPSS 27.0 .

Results: There were $36(60 \%)$ males and 24 (40\%) were females with mean age $48.14 \pm 7.58$ years in group A while in group B 39 (65\%) and 21 (35\%) patients were males and females with mean age $49.08 \pm 8.33$ years. Cirrhotic patients with red meat consumption $>60 \mathrm{gm} /$ day had high mortality rate as compared to patients with red meat consumption $<60 \mathrm{gm} /$ day $(10 \%$ Vs $3.33 \%)$ with $\mathrm{p}$-value 0.0001 .

Conclusion: A significant association of adverse outcomes was found in cirrhotic patients and red meat consumption.

Keywords: Chronic Liver Disease, Red Meat Consumption, Mortality
\end{abstract}

\section{INTRODUCTION}

In the developed countries, the intake of meat has increased [1.], and while several studies have suggested that the use of red-and-processed meat and oesophageal cancer, as well as the risk of processed meat and gum cancer, a thorough World Cancer Research Fund review concluded that evidence was limited and originated largely from case-control studies[2.].

Strong risk factors for CLD, such as aflatoxin exposure, increased alcohol use, and hepatitis $B$ virus (HBV) (HCV) chronic inflammation have been discovered (2). Such exposure can damage the hepatitis and lead to chronic liver illness (CLD). People with CLD are quite likely to die of their problems and are highly vulnerable to HCC development.[3] In patients without exposure to aflatoxins, alcohol, HBVs, or HCV, the significance of other risk factors is shown in a large proportion of HCC. [4,5]

Relatively few research have studied dietary connection with HCC risk [6-13] as opposed to several of the other malignancies. Most research conducted include a case-control design which evaluates dieting following HCC diagnosis, which can damage the health of individuals and may have an effect on dietary memory accuracy. Few research examined the association of white meat consumption with the risk of $\mathrm{HCC}$ and little or no information about the role of red or white meat in CLD was available.

The prospective National Institutes of Health (NIH)AARP (previously known as the American Association of Retired Persons) Diet and Health Study was shown to show favorable associations between red meat diet and liver cancer recently in order to analyze all cancers.[14] In this article we investigate different processes, as well as the likely function of white meat, underlying this connection. Red meat is the main dietary source of fatty acids that are saturated and monounsaturated. Deposition of fatty acids in the liver can cause non-alcoholic liver fatty diseases that can raise CLD and HCC risk.[15] Alternatively, high levels of heme iron are contained in red meat.[16] Persons with iron overload hemokromatosis have a significantly greater risk for liver cancer,[17,18] excess dieting iron in several parts of Africa contributes to the risk of CLD and HCC [1820], as well as phlebotomy and low iron Diets for chronic $\mathrm{HCV}$ (21) and may thus lower the risk for liver cancer. It is uncertain if moderate intakes of dietary iron have a part in developing CLD and HCC. We have done this study to examine the link between red meat consumption and chronic liver disease.

\section{MATERIALS AND METHODS}

This cross-sectional study was conducted atMedicine Department of Liaquat University Medical Hospital Jamshoro and Civil Hospital Khairpur Mir's for six months duration from December 2019 to November 2020and comprised of 120 patients. Patients' detailed demographics were recorded after taking informed written consent. Patients had cardiac disease and any other severe medical illness was excluded from this study.

Patients were aged between $18-60$ years with chronic liver disease were enrolled in this study. Patients were 
categorized in to two groups. Group A (red meat consumption $>60 \mathrm{gm} /$ day) and group B (red meat consumption $<60 \mathrm{gm} /$ day). Anthropometric measures were performed as otherwise stated in fasting conditions Liver ultrasound of all the patients was taken. A Magnetic Resonance Image (MRI) has been additionally carried out in standardized methods for each participant to quantify the fat and iron content of the liver as well as the hepatic volume. Outcomes in term of mortality between both groups were examined. All the data was analyzed by SPSS 27.0. Categorical variables were assessed by frequencies and percentages.

\section{RESULTS}

There were $36(60 \%)$ males and 24 (40\%) were females with mean age $48.14 \pm 7.58$ years in group A while in group B $39(65 \%)$ and 21 (35\%) patients were males and females with mean age $49.08 \pm 8.33$ years. (Table 1 )

Table 1: Baseline detailed demographics of enrolled cases

\begin{tabular}{|l|l|l|l|}
\hline Variables & Group A & Group B & Total \\
\hline Gender & $36(60 \%)$ & $39(65 \%)$ & 60 \\
\hline Male & $24(50 \%)$ & $21(355)$ & 60 \\
\hline Female & $48.14 \pm 7.58$ & $49.08 \pm 8.33$ & \\
\hline Mean Age (yrs) & & \\
\hline Diagnosis & & $28(56 \%)$ & 58 \\
\hline HCV & $30(50 \%)$ & $17(34 \%)$ & 32 \\
\hline HBV & $15(30 \%)$ & $8(16 \%)$ & 17 \\
\hline NAFLD & $9(18 \%)$ & $7(14 \%)$ & 13 \\
\hline HCC & $6(12 \%)$ &
\end{tabular}

Hospital stay was greater among the patients with red meat consumption $>60 \mathrm{gm} /$ day (group A) $13.05 \pm 5.12$ days as compared to the patients with meat consumption $<60$ $\mathrm{gm} /$ day (group B) $7.55 \pm 2.22$ days. Patients with red meat consumption $>60 \mathrm{gm} /$ day had high mortality rate as compared to patients with meat consumption $<60 \mathrm{gm} /$ day (10\% Vs $3.33 \%$ ) with p-value 0.0001. (table 2)

Table 2: Association of adverse outcomes among the patients of both groups

\begin{tabular}{|l|c|c|}
\hline Adverse outcomes & Group A & Group B \\
\hline Hospital stay (days) & $13.05 \pm 5.12$ & $7.55 \pm 2.22$ \\
\hline Mortality & $6(10 \%)$ & $2(3.33 \%)$ \\
\hline Yes & $56(90 \%)$ & $58(96.67 \%)$ \\
\hline No
\end{tabular}

\section{DISCUSSION}

This present study was conducted to determine the association of adverse outcomes in term of mortality in patients with cirrhosis and red meat consumption. In our study total 120 patients of both genders were presented, in which majority of the patients $65 \%$ were males. Patients were aged between $30-75$ years of age. We arranged to divide patients into two equal groups $A$ and $B$, group $A$ had patients of cirrhosis and group $B$ had patients without cirrhosis. Mean age of the patients in group $A$ and $B$ was (48.14 $\pm 7.58,49.08 \pm 8.33$ years). These finding were comparable to the previous studies. [8,9]

Majority $62.5 \%$ were males and $37.5 \%$ were females found in our study.[10] We found that most of the patients $48.33 \%$ had hepatitis C followed by HBV $26.67 \%, 14.67 \%$ patients had NAFLD and HCC was among $10.83 \%$. Our findings showed resemblance to the previous some studies.[11-14] Inconvenient dietary behavior is recognized as a risk factor for NAFLD[15] development. Failure to comply with healthy eating patterns and move to the west could play a significant role in NAFLD development [12]. The intake of foods characterizing Western dietary behaviours, such as soft drinks, fructose, meats and fast foods showed damaging health effects that promote obesity and related comorbidities, such as NAFLD[16,17]. Over the recent decade, several of the major risk factors for HCC have been changed, such as chronic hepatitis $B$ and hepatitis $\mathrm{C}$ viruses with directly acting antivirals, and the rate of HCCs associated with these variables is projected to decline. However, obesity and fatty liver are increasingly associated with $\mathrm{HCC}$, and the current epidemic of obesity cannot increase its frequency. Changes in nutrition and dietary patterns associated with increasing obesity may potentially have a direct impact on HCC disease. Studies such as reduced HCC risk by intake of yellow vegetable in patients with hepatitis B (HBV) or hepatitis C (HCV) infection have incorporated some of these mechanisms.[18]

In our study patients with meat consumption $>60 \mathrm{mg} /$ day resulted greater hospital stay $13.05 \pm 5.12$ days as compared to the patients with meat consumption $<60$ gm/day (group B) $7.55 \pm 2.22$ days. Previous research presented that the increased risk of chronic liver disease related with eating of meat.[19] The amount of rotten flake meat, which causes fibrosis and cirrhosis excessively, is considerable, even in haem iron [20,21]. The composition of red meat also contains high cholesterol and fat in relation to established cancer risk factors, including obesity, diabetes and metabolism. Furthermore, meat treatment and preservation generates possible carcinogenic substances, like N-nitrosocompounds[22]. In contrast, fish and chicken are less saturated in fat and cholesterol and higher in polyunsaturated fatty acids, which block the factor of tumour-necrosis and inflammation.[23]

Patients with red meat consumption $>60 \mathrm{gm} /$ day had high mortality rate as compared to patients with meat consumption $<60 \mathrm{gm} /$ day $(10 \%$ Vs $3.33 \%)$ with $p$-value 0.0001 .Different previous studies presented results that high consumption of meat ,ay results in liver fibrosis and advanced cirrhosis. Mortality rate among all these studies were comparable to our findings.[24,25] Cirrhotic patients with red meat eating have shown a strong combination of unfavorable effects.

\section{CONCLUSION}

A significant association of adverse outcomes was found in cirrhotic patients and red meat consumption.

\section{REFERENCES}

1. Shira Zelber-Sagi, Dana Ivancovsky-Wajcman, Naomi Flisslsakov, Muriel Webb , Dana Orenstein2, Oren Shibolet1,3 , RevitalKariv. High red and processed meat consumption is associated with non-alcoholic fatty liver disease and insulin resistance. Journal of Hepatology 2018 vol. 68 j 1239-1246

2. Recaredo G, Marin-Alejandre BA, Cantero I, et al. Association between Different Animal Protein Sources and Liver Status in Obese Subjects with Non-Alcoholic Fatty 
Liver Disease: Fatty Liver in Obesity (FLiO) Study. Nutrients. 2019;11(10):2359. Published 2019 Oct 3.

3. Liu X., Peng Y., Chen S., Sun Q. An observational study on the association between major dietary patterns and nonalcoholic fatty liver disease in Chinese adolescents. Medicine. 2018;97:1-7.

4. Nogueira M.A., Oliveira C.P., Ferreira Alves V.A., Stefano J.T., dos Reis Rodrigues L.S., Torrinhas R.S., Cogliati B., Barbeiro H., Carrilho F.J., Waitzberg D.L. Omega-3 polyunsaturated fatty acids in treating non-alcoholic steatohepatitis: A randomized, double-blind, placebocontrolled trial. Clin. Nutr. 2016;35:578-586.

5. Montella, M.; Crispo, A.; Giudice, A. HCC, diet and metabolic factors: Diet and HCC. Hepat. Mon. 2011, 11, 159

6. Soleimani D., Rezvani R., Goshayeshi L., Nematy M. Dietary patterns in relation to hepatic fibrosis among patients with nonalcoholic fatty liver disease. Diabetes Metab. Syndr. Obes. Targets Ther. 2019;12:315-324

7. Zelber-Sagi S., Salomone F., Mlynarsky L. The Mediterranean dietary pattern as the diet of choice for nonalcoholic fatty liver disease: Evidence and plausible mechanisms. Liver Int. 2017;37:936-949.

8. Fakhoury-Sayegh N., Younes H., Heraoui G.N.H.A., Sayegh $R$. Nutritional profile and dietary patterns of lebanese nonalcoholic fatty liver disease patients: A case-control study. Nutrients. 2017;9:1245.

9. Kurahashi, N.; Inoue, M.; Iwasaki, M.; Tanaka, Y.; Mizokami, M.; Tsugane, S.; for the JPHC Study Group. Vegetable, fruit and antioxidant nutrient consumption and subsequent risk of hepatocellular carcinoma: A prospective cohort study in Japan. Br. J. Cancer 2009, 100, 181-184

10. Freedman N.D., Cross A.J., McGlynn K.A., Abnet C.C., Park Y., Hollenbeck A.R., Schatzkin A., Everhart J.E., Sinha R.
Association of meat and fat intake with liver disease and hepatocellular carcinoma in the NIH-AARP cohort. J. Natl. Cancer Inst. 2010;102:1354-1365.

11. Polesel, J.; Talamini, R.; Montella, M.; Maso, L.; Crovatto, M.; Parpinel, M.; Franceschi, S.; Izzo, F.; Tommasi, L.G.; Serraino, D.; et al. Nutrients intake and the risk of hepatocellular carcinoma in Italy. Eur. J. Cancer 2007, 43, 2381-2387

12. Bonkovsky, H. Iron and the Liver. Am. J. Med. Sci. 1991 , 301, 32-43

13. Luo, J.; Yang, Y.; Liu, J.; Lu, K.; Tang, Z.; Liu, P.; Liu, L.; Zhu, Y. Systematic review with meta-analysis: Meat consumption and the risk of hepato-cellular carcinoma. Aliment. Pharm. 2014, 39, 913-922

14. Endres, S.; Ghorbani, R.; Kelley, V.; Georgilis, K.; Lonnemann, G.; van der Meer, J.; Cannon, J.G.; Rogers, T.S.; Klempner, M.S.; Weber, P.C.; et al. The Effect of Dietary Supple-mentation with $\mathrm{n}-3$ Polyunsaturated Fatty Acids on the Synthesis of Interleukin-1 and Tumor Necrosis Factor by Mononuclear Cells. N. Engl. J. Med. 1989, 320, 265-271

15. Tamura, T.; Wada, K.; Konishi, K.; Goto, Y.; Mizuta, F.; Koda, S.; Hori, A.; Tanabashi, S.; Matsushita, S.; Tokimitsu, N.; et al. Coffee, Green Tea, and Caffeine Intake and Liver Cancer Risk: A Prospective Cohort Study. Nutr. Cancer 2018, 70, 1210-1216

16. Modares Mousavi S.R., Geramizadeh B., Anushiravani A., Ejtehadi F., Anbardar M.H., Moini M. Correlation between Serum Ferritin Level and Histopathological Disease Severity in Non-alcoholic Fatty Liver Disease. Middle East J. Dig. Dis. 2018;10:90-95. 